\section{Comment on: "Tumor Aggressiveness and Patient Outcome in Cancer of the Pancreas Assessed by Dynamic ${ }^{18}$ F-FDG PET/CT"}

TO THE EDITOR: We read with great interest the article by Ron Epelbaum et al. (1) on the use of dynamic ${ }^{18}$ F-FDG PET/CT to assess tumor aggressiveness and overall outcome in patients with pancreatic cancer. The authors have successfully shown how quantitative parameters of tracer kinetics can add value to ${ }^{18}$ F-FDG PET imaging. They also sensibly speculate about the potential capabilities of dynamic ${ }^{18} \mathrm{~F}$-FDG PET as an evolving strategy that may, in the future, enhance the accuracy of pretreatment risk stratification and become integrated into prognostic scores for individualized treatment tailoring. On the other hand, as mentioned by the authors, quantitative dynamic PET analysis is currently considered a cumbersome technique with value demonstrated mainly in research settings. Although it is expected that the modeling component of this approach will be simplified for future clinical use, the nature of extensive data acquisition by this technique seems not to be altered significantly, since it is determined mostly by ${ }^{18}$ F-FDG kinetics and tumor biology. For this reason, the protracted acquisition time will still limit widespread application of this valuable method in routine practice outside major academic centers. As mentioned by the authors, several studies have found that standardized uptake value (SUV) measured by static ${ }^{18}$ F-FDG PET scans was an independent predictive factor for overall survival in the multivariate analysis (2). Similarly, in this study $\mathrm{SUV}_{1}$ (early) and $\mathrm{SUV}_{2}$ (late) were predictors of overall survival in the univariate model; however, as was predictable, these factors were not significant predictors of survival in the multivariate model, which included ${ }^{18} \mathrm{~F}$-FDG kinetic parameters.

The first of 3 comments on this article is about the correlations between SUV and ${ }^{18}$ F-FDG kinetic parameters. Previous studies have shown correlations between early and late SUVs of ${ }^{18} \mathrm{~F}-\mathrm{FDG}$ PET imaging and transmembrane glucose transporters and hexokinase expression in pancreatic (3) and other tumor cells (4). Likewise, ${ }^{18} \mathrm{~F}$-FDG kinetic parameters including $K_{1}$ and $k_{2}$ are indicators of transmembrane transport of ${ }^{18} \mathrm{~F}-\mathrm{FDG}$, and $k_{3}$ and $k_{4}$ are indicators of intracellular ${ }^{18} \mathrm{~F}$-FDG phosphorylation and dephosphorylation, respectively. Therefore, SUVs and kinetic parameters and their derivatives, such as global influx of ${ }^{18} \mathrm{~F}-\mathrm{FDG}$ and retention index $\left(\left[\left(\mathrm{SUV}_{2}-\mathrm{SUV}_{1}\right) / \mathrm{SUV}_{1}\right]\right)$, have intrinsic correlations, which raise concerns about the potential multicollinearity between them when they are applied as independent explanatory variables in regression models (5). In the current study, as was briefly noted by the authors, the highly predictive significance of kinetic parameters covered the role of $\mathrm{SUV}_{1}$ and $\mathrm{SUV}_{2}$ in the multivariate survival analysis. Early and late SUVs and ${ }^{18} \mathrm{~F}$-FDG kinetic parameters are indicators of glucose metabolism - the actual biologic explanatory cause; therefore, it seems statistically reasonable to choose ${ }^{18}$ F-FDG kinetic parameters since these values have a smaller degree of random error (6). However, it is not cost-effective for most imaging centers to

COPYRIGHT (C) 2014 by the Society of Nuclear Medicine and Molecular Imaging, Inc. assign their PET facilities to time-consuming dynamic PET imaging. In addition, a significant proportion of patients cannot tolerate remaining motionless while in the gantry of PET/CT scanners for an extended time (more than $60 \mathrm{~min}$ in this study). Considering these facts, we believe a piece of clinically important data was not reported in this article and that it would be valuable for the authors to conduct a multivariate survival analysis after excluding kinetic parameters to clarify the value of SUVs in this group of patients. This new analysis may hopefully develop clear cutoffs for early and late SUV measurements, which can be applied as practical predictive factors of overall survival in the clinical setting.

The second comment is related to parameters that can be retrieved from dual- or multiple-time-point PET studies. In some studies on dual-time-point imaging, it has been speculated that measuring retention indices of SUVmax could overcome many factors that limit the value of SUVmax measurements, including blood glucose levels and body weight $(7,8)$. These studies also demonstrated the added value of calculating the retention index in prognostic models. Although $\mathrm{SUV}_{1}$ and $\mathrm{SUV}_{2}$ could not significantly predict long-term survival, retention indexes were independent predictive factors on the Cox regression model $(7,8)$. Therefore, we believe it would be productive for the authors to calculate the retention index of SUVmax and use it in univariate and multivariate analysis. However, involving retention index in the multivariate analysis requires that the possibility of multicollinearity between variables be considered again.

The third comment is related to ${ }^{18} \mathrm{~F}$-FDG PET parameters that can be used for predicting progression-free and overall survival. Some studies performed on patients with various types of cancer suggested that volume-based PET parameters such as metabolic tumor volume (MTV) and total lesion glycolysis (TLG = SUVmean $\times$ MTV) may predict overall survival whereas SUVmax alone is not an optimal predictive factor $(9,10)$. Hence, measuring and incorporating MTV and TLG in the survival models may add more remarkable aspects to this study. However, in addition to multicollinearity issues in the statistical analysis, partial-volume correction methods should be considered to measure these parameters precisely (11).

In conclusion, we believe that calculation and head-to-head comparison of all prospective imaging biomarkers in dynamic PET studies, including estimated SUV thresholds, retention indices, TLG, MTV, and kinetic parameters, would better elucidate the correlation among these factors and may provide further valuable strategies for future investigations and routine practice.

\section{REFERENCES}

1. Epelbaum R, Frenkel A, Haddad R, et al. Tumor aggressiveness and patient outcome in cancer of the pancreas assessed by dynamic ${ }^{18} \mathrm{~F}$-FDG PET/CT. $J$ Nucl Med. 2013;54:12-18.

2. Schellenberg D, Quon A, Minn AY, et al. 18Fluorodeoxyglucose PET is prognostic of progression-free and overall survival in locally advanced pancreas cancer treated with stereotactic radiotherapy. Int J Radiat Oncol Biol Phys. 2010;77:1420-1425.

3. Higashi T, Saga T, Nakamoto Y, et al. Relationship between retention index in dual-phase ${ }^{18} \mathrm{~F}-\mathrm{FDG}$ PET, and hexokinase-II and glucose transporter-1 expression in pancreatic cancer. $J$ Nucl Med. 2002;43:173-180. 
4. Nakajo M, Kajiya Y, Tani A, Yoneda S, Shirahama H, Higashi M. ${ }^{18}$ FDG PET for grading malignancy in thymic epithelial tumors: significant differences in ${ }^{18}$ FDG uptake and expression of glucose transporter- 1 and hexokinase II between low and high-risk tumors-preliminary study. Eur J Radiol. 2012;81: $146-151$.

5. Van Steen K, Curran D, Kramer J, et al. Multicollinearity in prognostic factor analyses using the EORTC QLQ-C30: identification and impact on model selection. Stat Med. 2002;21:3865-3884.

6. Doot RK, Kurland BF, Kinahan PE, Mankoff DA. Design considerations for using PET as a response measure in single site and multicenter clinical trials. Acad Radiol. 2012;19:184-190.

7. Houseni M, Chamroonrat W, Zhuang J, Gopal R, Alavi A, Zhuang H. Prognostic implication of dual-phase PET in adenocarcinoma of the lung. J Nucl Med. 2010;51:535-542.

8. Lyshchik A, Higashi T, Nakamoto Y, et al. Dual-phase ${ }^{18}$ F-fluoro-2-deoxy-Dglucose positron emission tomography as a prognostic parameter in patients with pancreatic cancer. Eur J Nucl Med Mol Imaging. 2005;32:389-397.

9. Lim R, Eaton A, Lee NY, et al. ${ }^{18}$ F-FDG PET/CT metabolic tumor volume and total lesion glycolysis predict outcome in oropharyngeal squamous cell carcinoma. J Nucl Med. 2012;53:1506-1513.

10. Fonti R, Larobina M, Del Vecchio S, et al. Metabolic tumor volume assessed by ${ }^{18} \mathrm{~F}$-FDG $\mathrm{PET} / \mathrm{CT}$ for the prediction of outcome in patients with multiple myeloma. J Nucl Med. 2012;53:1829-1835.

11. Basu S, Kwee TC, Torigian D, Saboury B, Alavi A. Suboptimal and inadequate quantification: an alarming crisis in medical applications of PET. Eur J Nucl Med Mol Imaging. 2011;38:1381-1382.

\section{Ali Salavati \\ Babak Saboury \\ Abass Alavi* \\ *Hospital of the University of Pennsylvania \\ 3400 Spruce St., 1 Donner Building \\ Philadelphia, PA 19104-4283 \\ E-mail: abass.alavi@uphs.upenn.edu}

Published online Dec. 9, 2013

DOI: 10.2967/jnumed.113.130138

REPLY: We would like to thank Dr. Salavati and his coauthors for the interesting comment on our study (1). As they mentioned, dynamic PET and PET/CT are more time-consuming and at the moment are therefore confined to research projects for scientific purposes. Furthermore, dynamic PET/CT requires dedicated evaluation software. However, the introduction of new-generation PET/CT scanners has reduced the total acquisition time because of, for example, new detector materials such as lutetium oxyorthosilicate, which improves the counting rate performance, as well as 3-dimensional acquisition protocols. Moreover, new-generation PET/CT scanners also allow dynamic (list-mode) multibed acquisitions. In the future, this technologic improvement will allow for dynamic partial-body PET/CT studies without the need for additional bed positions in static mode, with a shorter acquisition than in our study (2). We agree that an additional limitation hampering the use of dynamic protocols in a clinical environment is the lack of operator-friendly and robust evaluation software-an omission that will hopefully be addressed by manufacturers. The existing software for calculation of transport rates is based on a 2-tissuecompartment model for oncologic studies. This software is not robust enough because it is based on an iterative fitting, like the Levenberg-Marquardt algorithm. We presented a solution that is based on the use of an oncologic reference database and a support vector machine algorithm (3). Routine use of dynamic PET/CT requires that the calculated rates be reproducible - a problem that should be solved in the future.
Ludwig Strauss proposed at the end of the 1980s the use of the standardized uptake value (SUV) as a robust value that can easily be calculated for the evaluation of PET data (4). SUVs are widely used and lead to good results, provided that the values are acquired under standardized conditions, such as at a defined time point after tracer injection, with glucose levels within the normal range, and with the same reconstruction algorithms. John W. Keyes, Jr., wrote an interesting paper in The Journal of Nuclear Medicine in 1995 titled "SUV: Standard Uptake or Silly Useless Value?" In this paper he doubted the usefulness of SUV and discussed the limitations of this semiquantitative approach in detail (5). Nineteen years later, everybody uses the SUV or its derivatives (such as SUVmax, SUVlean, or even total lesion glycolysis) as a first quantitative approach. It remains to be seen how silly or useless dynamic multibed PET/CT (including parametric imaging) in oncology will be in the future.

Dynamic imaging allows the registration of tracer kinetics over time instead of at only one time point after the tracer injection as static images do. Pharmacokinetic studies are helpful not only for the evaluation of new tracers but also for the evaluation of small therapeutic effects, such as the use of ${ }^{18}$ F-FDG early after the onset of chemotherapy. Furthermore, the use of kinetic parameters may help to differentiate between benign and less aggressive tumors (e.g., lipomas from low-grade liposarcomas) (6). In a recent paper, we demonstrated a correlation between $k_{1}$ and angiogenesis-related genes (7). Based on dynamic datasets, parametric imaging can be applied using different algorithms. Parametric images allow the visualization of dedicated parameters of radiopharmaceutical kinetics, such as perfusion, transport, or phosphorylation in the case of ${ }^{18} \mathrm{~F}-\mathrm{FDG}$. Karakatsanis et al. recently presented some aspects of the use of whole-body PET parametric imaging and, for example, Patlak analysis in addition to SUVs for tumor diagnosis and therapy response monitoring (8).

We agree that several approaches available today may be used for the evaluation of oncologic ${ }^{18} \mathrm{~F}$-FDG imaging, including metabolic tumor volume and total lesion glycolysis. We decided to use an analysis based primarily on the pharmacokinetic data, and this proved to be successful. We hope our colleagues will succeed as well in future using any other approach they may wish to choose.

\section{REFERENCES}

1. Epelbaum R, Frenkel A, Haddad R, et al. Tumor aggressiveness and patient outcome in cancer of the pancreas assessed by dynamic ${ }^{18} \mathrm{~F}-\mathrm{FDG}$ PET/CT. J Nucl Med. 2013;54:12-18.

2. Dimitrakopoulou-Strauss A, Pan L, Strauss LG. Quantitative approaches of dynamic FDG-PET and PET-CT studies (dPET/CT) for the evaluation of oncological patients. Cancer Imaging. 2012;12:283-289.

3. Pan L, Mikolajczyk K, Strauss L, Haberkorn U, Dimitrakopoulou-Strauss A. Machine learning based parameter imaging and kinetic modeling of PET data [abstract]. J Nucl Med. 2007;48(suppl):158P.

4. Strauss LG, Conti PS. The applications of PET in clinical oncology. J Nucl Med. 1991;32:623-648.

5. Keyes JW, Jr. SUV: standard uptake or silly useless value? J Nucl Med. 1995;36: 1836-1839.

6. Dimitrakopoulou-Strauss A, Strauss LG, Schwarzbach M, et al. Dynamic PET ${ }^{18} \mathrm{~F}$-FDG studies in patients with primary and recurrent soft-tissue sarcomas: impact on diagnosis and correlation with grading. J Nucl Med. 2001;42:713-720.

7. Strauss LG, Koczan D, Klippel S, et al. Impact of angiogenesis-related gene expression on the tracer kinetics of ${ }^{18}$ F-FDG in colorectal tumors. J Nucl Med. 2008;49:1238-1244.

8. Karakatsanis NA, Lodge MA, Zhou Y, Wahl RL, Rahmin A. Dynamic whole-body PET parametric imaging: II. Task-oriented statistical evaluation. Phys Med Biol. 2013;58:7419-7445. 